\title{
Projeto de um sensor de posição utilizando webcam para um sistema mesa bola
}

\author{
Sabrina Loiola de Morais ${ }^{[1]}$, Marcelo Roberto Bastos Guerra Vale ${ }^{[2]}$ \\ [1] Universidade Federal Rural do Semi Árido; sabrinaloiola9@gmail.com \\ ${ }^{[2]}$ Universidade Federal Rural do Semi Árido; marceloguerra@ufersa.edu.br \\ Recebido: 07/07/2020; \\ Aceito: 09/08/2020; \\ Publicado: 11/09/2020.
}

Resumo: O constante interesse em técnicas de processamento digital de imagens (PDI) e sua importância em diversas áreas de atuação, tem impulsionado estudos que envolvam implementações práticas. Este trabalho tem o intuito de utilizar uma câmera web como um sensor de posição para um sistema de controle mesa bola. Esse projeto baseia-se nas técnicas de PDI, utilizando-se do software Matlab ${ }^{\mathrm{TM}}$ em conjunto com a câmera. Assim como discorre sobre os materiais utilizados e suas respectivas funções e especificações. Aborda também as técnicas utilizadas para obtenção dos resultados. Os resultados apresentados se mostraram satisfatórios, indicando o bom funcionamento do conjunto e o valor posição da bola muito próximo ao real.

Palavras-chave: PDI, câmera, mesa-bola.

Abstract: The advance of techniques about digital image processing (DIP) and its importance on the most varied fields, have been pushing researches about practical implementation of this subject. This paper has the purpose of using a webcam as a position sensor for a ball-plate system. This project is based on DIP techniques, using Matlab $^{\mathrm{TM}}$ software co-working with the webcam. As describe about the materials used and its functions and abric especifications. Also addresses the result's techniques and the way to found them. The results showed on this paper were satisfactory, displaying a good operation of the entire system and also a very close to real ball's position.

Key-words:DIP(digital image processing), webcam, ball-plate.

\section{INTRODUÇÃO}

S egundo [1], as áreas de processamento de imagens e visão por computador vêm apresentando expressivo $\mathrm{S}$ desenvolvimento nas últimas décadas. Tal crescimento pode ser detectado na área acadêmica, onde o assunto é objeto de pesquisas, teses e dissertações nas mais importantes universidades brasileiras e mundiais.

Ainda segundo Marques Filho, sendo explorado em uma infinita gama de aplicações, o processamento digital de imagens (PDI) pode ser encontrado desde os aspectos mais triviais do cotidiano, como em fotografias e tratamento de imagens, quanto aos mais complexos, utilizados por grandes indústrias e pela medicina. Como 
tomografias computadorizadas, radiografias, inspeção industrial e ainda com aplicações militares na utilização em radares e observações astronômicas.

Uma das aplicações do PDI é o controle e/ou detecção de posição de objetos, utilizando-se de uma câmera como um sensor sensível a movimentos. De posse dos dados, estes seriam aplicados posteriormente como entrada de um sistema de controle ou ainda para outros tipos de análises como vigilância de locais, uso em cirurgias delicadas, entre demais aplicações.

O nível de precisão e o tipo desses sensores são proporcionais ao estilo de aplicação em que irão ser utilizados, variando também com o tipo de ambiente, iluminação e a necessidade da velocidade de captura de dados.

Este trabalho tem o objetivo de explanar detalhadamente, desde a base teórica até a construção e implementação de uma câmera web como sensor para um sistema de controle mesa bola.

Para detecção da posição da bola em tempo real foi desenvolvido um algoritmo de visão computacional no software Matlab ${ }^{\mathrm{TM}}$, utilizando-se da biblioteca de aquisição de imagens e de ferramentas de reconhecimento de objetos.

\subsection{Objetivo Geral}

Este trabalho tem o intuito de utilizar uma câmera web (caracterizada por ser uma câmera de vídeo de baixo custo) como um sensor de posição para um sistema de controle mesa bola. O objetivo do sensor é que seja aplicável em qualquer superfície, de fácil ajuste para o usuário e que seja rápido o suficiente - visto que o sistema de controle a ser aplicado apresenta dinâmica rápida.

\subsection{Objetivos Específicos}

- Construção da estrutura física do sistema;

- Configuração da câmera e aplicação de técnicas de processamento digital de imagens para detecção da bola;

- Identificar apenas objetos redondos;

- Retornar a posição do centro da bola em tempo real;

- Calibração do sensor (câmera web) em relação à plataforma de controle;

\section{MATERIAIS E MÉTODOS}

Nesta seção são explicados os principais componentes do sistema, assim como os métodos utilizados para aquisição das imagens e seu processamento.

\subsection{Materiais}

Na Figura 1 estão apresentados todos os componentes do sistema e suas respectivas posições em uma vista isométrica. 


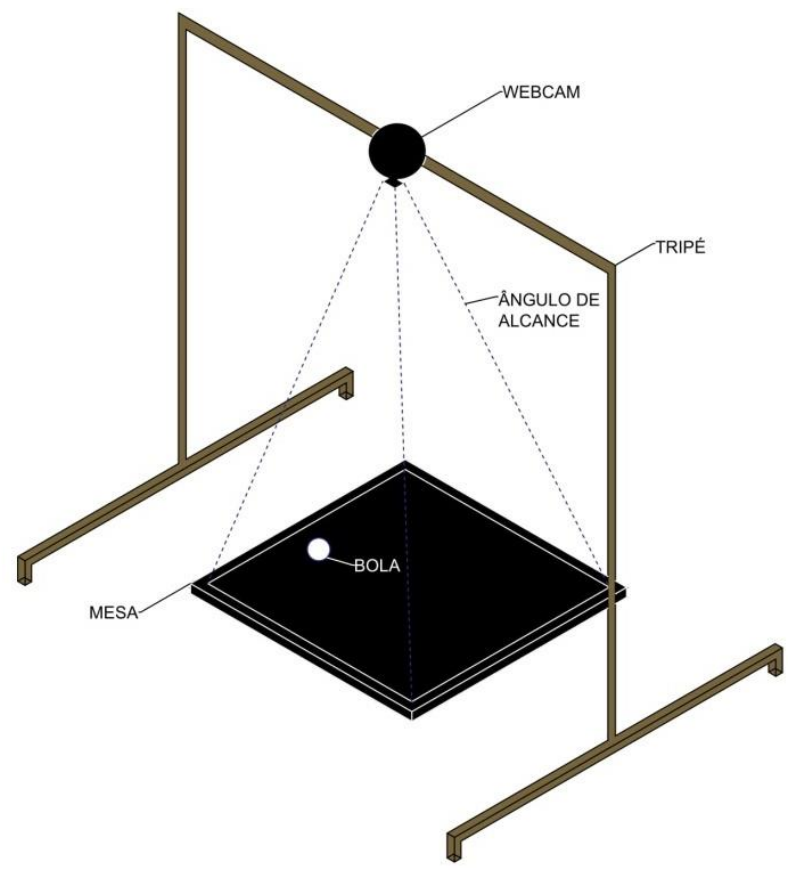

FiguRA 1. Sistema Simulado (autoria própria, 2019)

A Figura 1 apresenta o tripé com a câmera acoplada e direcionada de forma quase perpendicular à mesa, obtendo assim o melhor ângulo de captação da imagem. Já na Figura 2, se tem a imagem do sistema real montado.

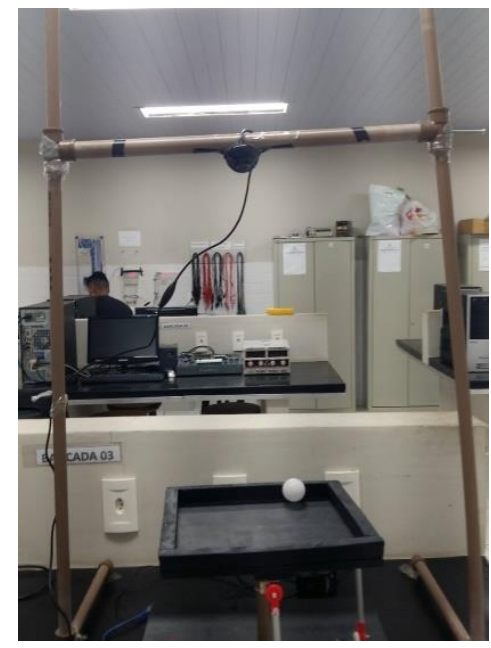

FIGURA 2. Sistema Real (autoria própria, 2019)

Nas subseções a seguir serão detalhados cada componente do sistema.

\subsubsection{Câmera}

Foi adquirida uma câmera webcam para servir como sensor do sistema, se aproveitando do fato de que o software MATLAB ${ }^{\mathrm{TM}}$ tem uma biblioteca específica para esse tipo de câmera, como ilustrado na Figura 3. 


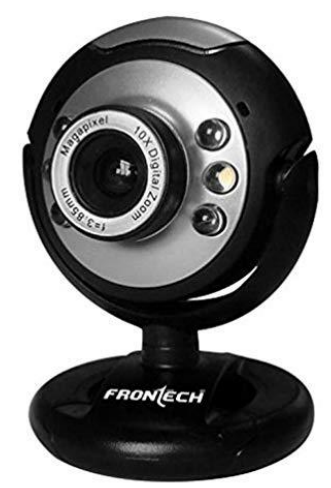

FIGURA 3. Câmera Utilizada (autoria própria, 2019)

A câmera em questão tem capacidade máxima de resolução de até 640x480 e mínima de 160x120 pixels.

\subsubsection{Tripé}

Para que fosse possível obter um bom posicionamento da câmera (de forma que essa ficasse diretamente sobre o tablado, evitando interferências na aquisição da imagem), foi construído um tripé de canos de policloreto de vinilha (PVC), como ilustrado na Figura 1.

O tripé conta com altura e deslocamento vertical reguláveis para que seja possível ajustar a posição da câmera sem que fosse interferida na posição da plataforma.

\subsubsection{Mesa}

A mesa utilizada como base para o sistema está ilustrada na Figura 4.

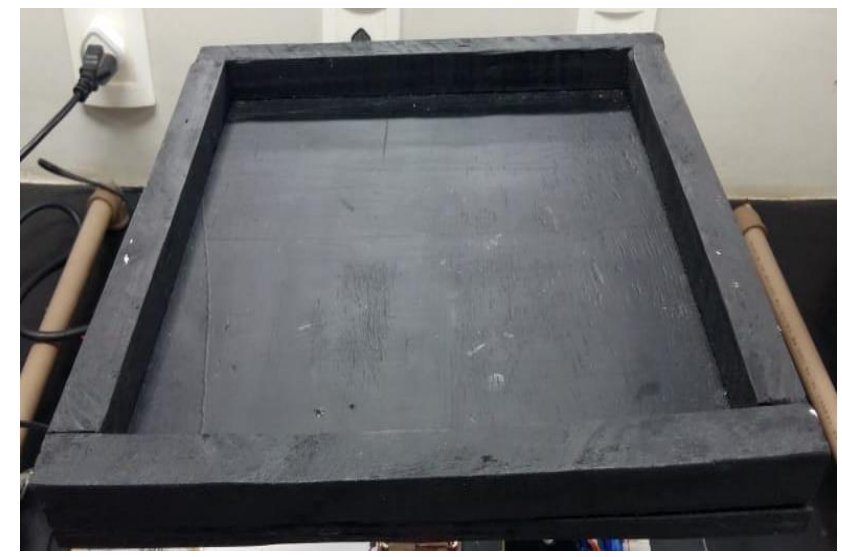

FIGURA 4. Mesa Utilizada (autoria própria, 2019)

Tomando como base um sistema de coordenadas x,y convencional, a mesa conta com medidas internas de 25 centímetros no eixo x e 26.5 centímetros no eixo y.

\subsubsection{Bola}

A bola utilizada no sistema está ilustrada na Figura 5. 


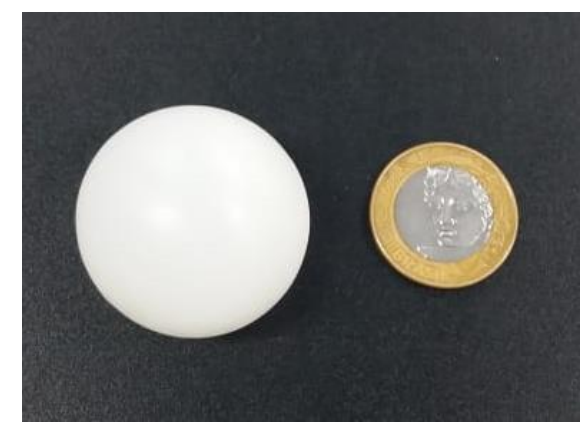

FIGURA 5. Bola Utilizada (autoria própria, 2019)

A bola utilizada aparece na Figura 5 ao lado de uma moeda, para comparação do seu tamanho. Foi utilizada uma bola de peso leve, aproximadamente 2,4 gramas, para que o momento inercial não fosse grande, $o$ que poderia atrapalhar no desempenho do sistema.

\subsection{Método}

Para uma melhor associação do sistema apresentado nesse trabalho, na Figura 6 mostra-se os passos no processo de PDI do sistema usado.

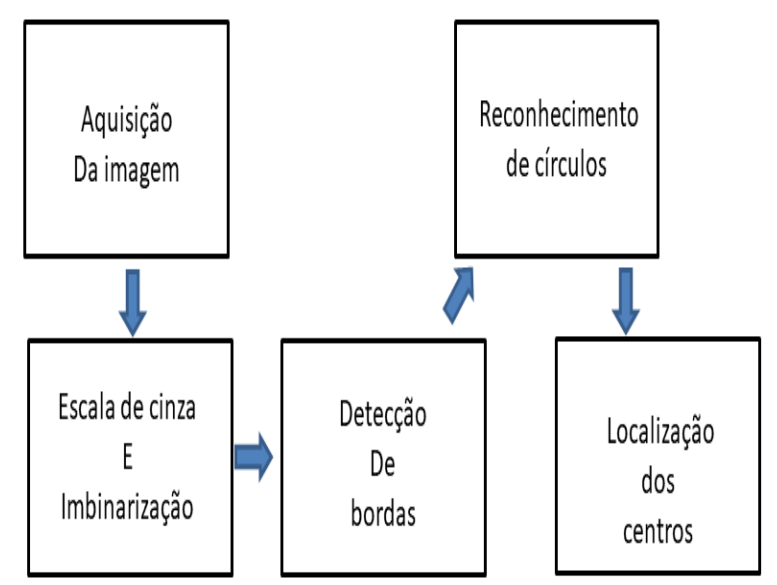

FIGURA 6. Passos no processo de PDI usado no sistema mesa bola (autoria própria, 2019)

Em um primeiro momento, haverá a captura da imagem pela biblioteca de aquisição de imagens do software Matlab ${ }^{\mathrm{TM}}$ em conjunto com a webcam, que permitirá a localização da bola em tempo real. Depois haverá a conversão da imagem para preto e branco e logo depois para binário [2] para que o processo morfológico seja otimizado, dando maior contraste entre bola e mesa. Em seguida, haverá o processo morfológico que se dará pela erosão e extração de fronteiras da imagem, permitindo que as bordas contidas na imagem sejam identificadas.

Dando continuidade, haverá o processo de segmentação que constará na identificação dos círculos e de seus respectivos centros [3], utilizando [4]. Dado o diâmetro da bola utilizada, foi colocado um raio variante de cinco a trinta como parâmetro de entrada.

Para cálculo da posição real da bola, foi medido o espaço de vídeo que a câmera mostrava (ângulo de alcance) com origem no canto superior direito, como ilustra a Figura 7, pelo número de pixels de resolução em que a câmera se encontrava (160x120). 


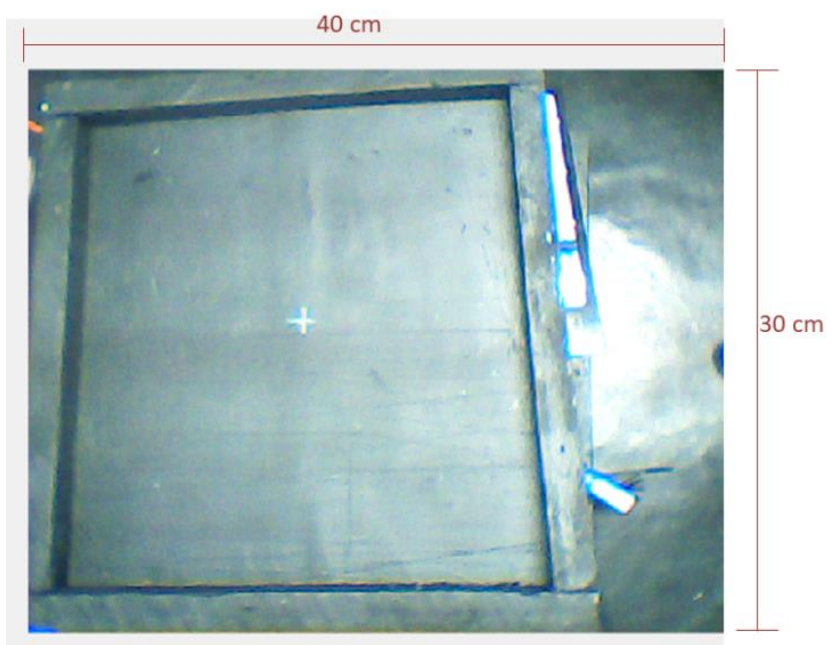

FIGURA 7. Ângulo de alcance da câmera (autoria própria, 2019)

Esse processo foi necessário, pois se a câmera ficasse muito baixa, não observaria o tablado todo e existiriam áreas em branco sem cobertura da câmera.

Os processos morfológicos e de segmentação foram feitos utilizando a função 'imfindcircles' [5] do software Matlab ${ }^{\mathrm{TM}}$, que identificava apenas os centros dos círculos de cor mais clara que o fundo. Essa função permite também encontrar objetos mais claros que o fundo, sendo assim de fácil adaptação para outros ambientes.

Foi feito ainda um laço específico no programa para que fosse avisado quando a câmera não conseguisse captar a bola, ou seja, possíveis pontos cegos do sistema ou quando a bola estivesse se movendo em grande velocidade sobre a mesa. A condição colocada foi que quando a câmera não estivesse localizando a bola, uma mensagem seria exibida e o sistema usaria o último valor de iteração como valor atual.

\section{RESULTADOS}

Após o procedimento de montagem e certificando-se que todos os componentes se encontravam em seus respectivos lugares, foram realizadas medições de modo a certificar o funcionamento do sistema.

$\mathrm{Na}$ Figura 8, ilustra-se a imagem de retorno do software, identificando a bola com um círculo azul e sua posição em relação à mesa no canto superior esquerdo - em vermelho a posição no eixo x e em azul a posição no eixo y. 

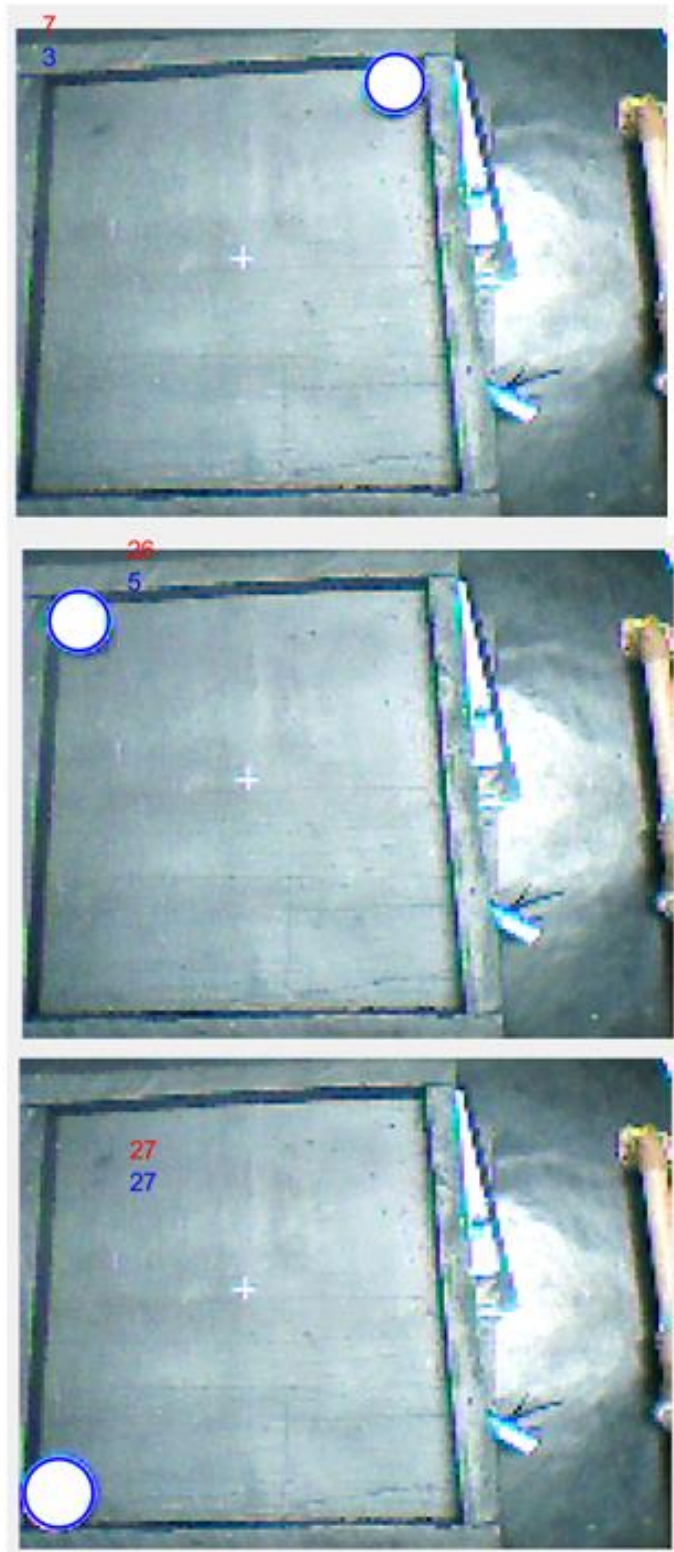

FIGURA 8. Posição da bola sobre a mesa (autoria própria, 2019)

O tempo de amostragem de cada iteração da posição do centro dos círculos foi cerca de 0,4 segundos mostrando-se compatível e rápido o suficiente para o sistema mesa-bola.

A primeira posição de centro da bola no eixo x é quatro centímetros e a última é (vinte e seis). Já no eixo y, a primeira posição também é quatro e a última é (vinte e sete) centímetros.

Foi observado que o valor retornado pelo software era bem próximo do real, mas que ainda eram necessários alguns ajustes na função de posição - que até então apresentava puramente o valor retornado, sem nenhuma modificação. Esses ajustes foram feitos pela calibração da câmera, explanada no item a seguir.

\subsection{Calibração}

Para melhor desempenho do sistema e ajuste fino do valor retornado pelo software foi feita a medição centímetro a centímetro da posição da bola na mesa. Movendo a bola no passo de um centímetro e observando a localização do centro dada pela câmera, foi feita a comparação entre o valor esperado e o retornado pela câmera e feita uma linearização dessa diferença, como apresentado na Figura 9. 


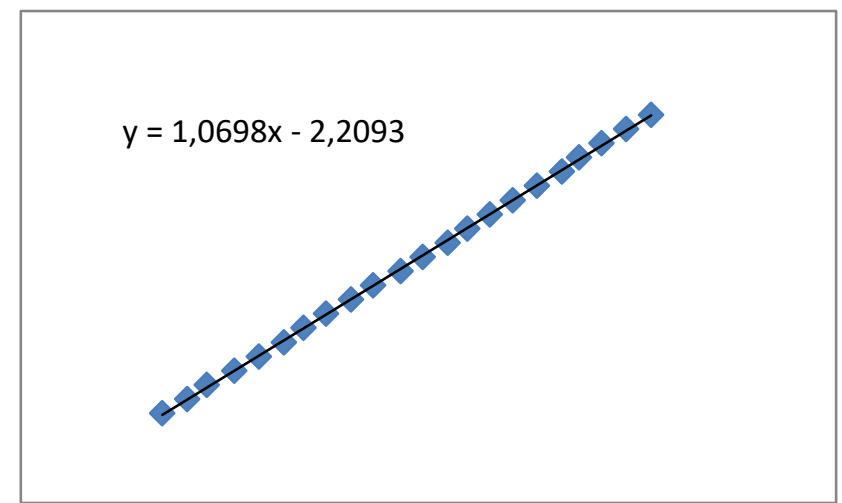

FIGURA 9. A. Parametrização no eixo x (autoria própria, 2019)

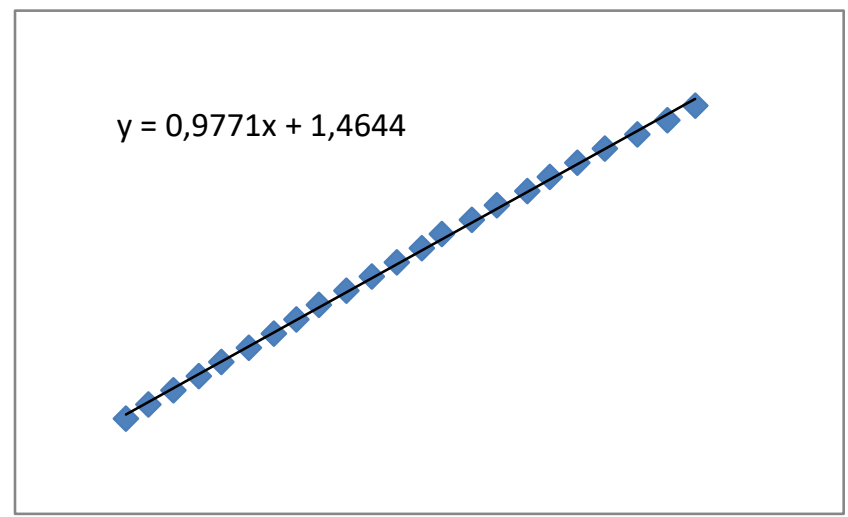

FIGURA 9.B. Parametrização no eixo x (autoria própria, 2019)

$\mathrm{Na}$ linearização, foi retornado os valores de funções expressado nas Fig. 9 A e 9B, essa diferença foi utilizada no código de programação utilizado, corrigindo um possível erro de localização da bola. O resultado da posição da bola já com parametrização está ilustrado na Figura 10. 

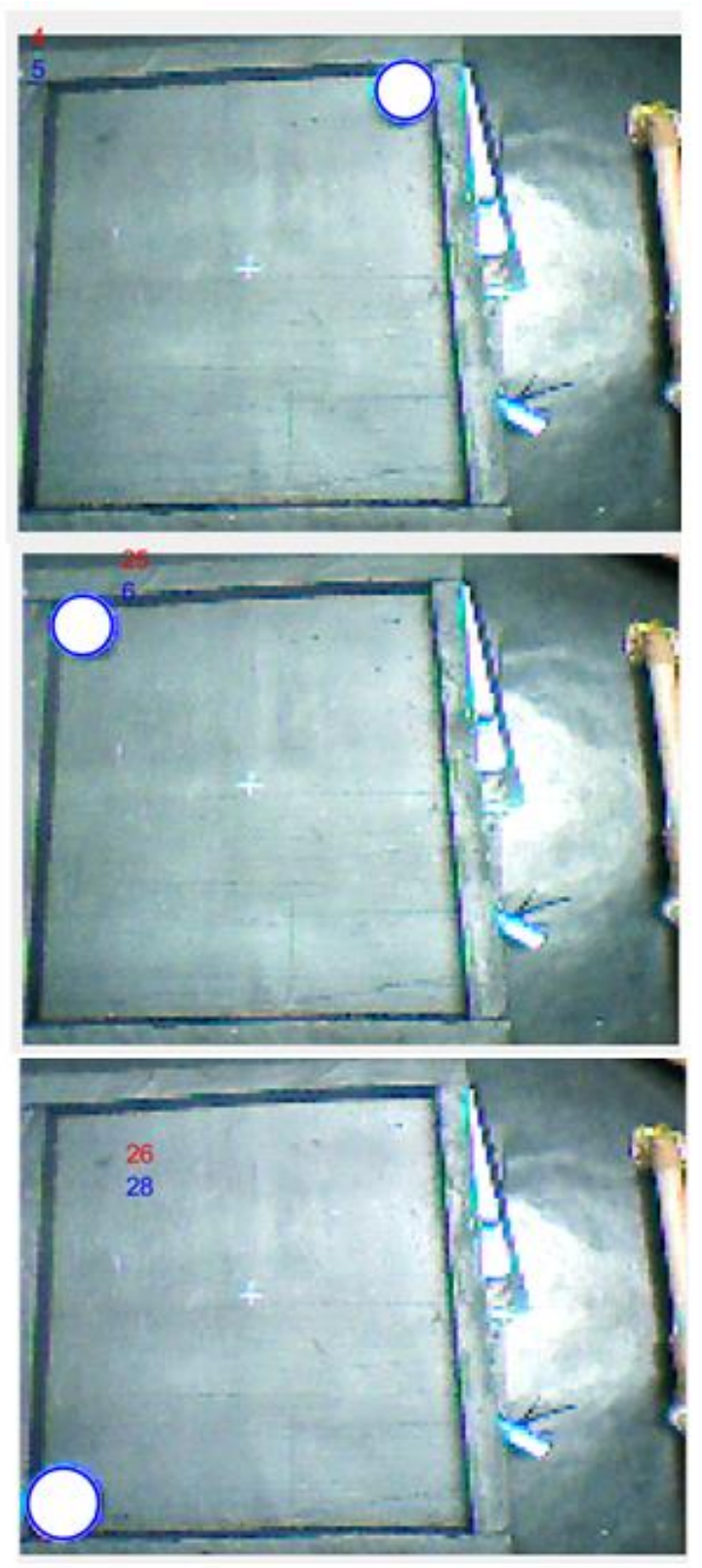

FIGURA 10. Posição da bola sobre a mesa com calibração (autoria própria, 2019)

Com os ajustes da calibração, os resultados observados foram melhores, indicando valores mais próximos dos reais do que os observados na Figura 9, caracterizando assim o bom desempenho do sistema.

Na Figura 11, é possível observar outro objeto circular e um não circular junto com a bola na mesa. 


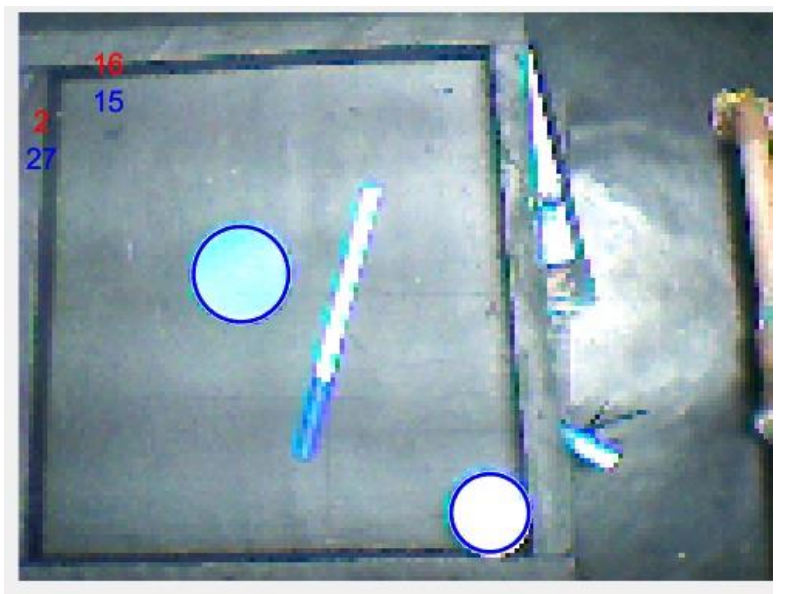

FIGURA 11. Objetos sobre a mesa (autoria própria, 2019)

Como ilustrado na Figura 11, os objetos circulares foram identificados e o objeto não circular não foi localizado pela câmera, mostrando o bom desempenho em identificar apenas objetos circulares. Foi analisado ainda que o sistema apresentou um comportamento adequado com as luzes do laboratório ligadas, o que exclui problemas de reflexo que poderiam vir a atrapalhar o desenvolvimento.

\section{CONCLUSÃO}

O trabalho elaborado apresentou uma proposta de um sensor para um sistema de controle mesa-bola utilizando uma webcam, de modo a estudar e aperfeiçoar técnicas de processamento digital de imagens combinadas ao controle digital. Desta forma, o trabalho foi desenvolvido e testado em laboratório e mostrou resultados satisfatórios, atendendo às expectativas de medição de posição, comportamento sob luz forte e controle de tempo de cada iteração. O sistema é de fácil entendimento e utiliza seus componentes aliados a um processamento digital de natureza descomplicada. Assim, é possível afirmar que o sistema é confiável e pode ser usado em diversos tipos de ambientes, não sendo atrapalhado pela iluminação forte nem pela cor dos objetos e/ou fundo. Esse trabalho é apenas um sistema inicial e fica aberto para trabalhos futuros e quaisquer melhorias, como o acréscimo de funções com o objetivo de ultrapassar obstáculos, realizar movimentos pré programados ou melhorar seu funcionamento de alguma maneira.

\section{REFERÊNCIAS}

[1] MARQUES FILHO, Ogê; NETO, Hugo Vieira. Processamento digital de imagens. Brasport, 1999.

[2] MATLAB -Imbinarização, 2010. [Internet] Disponível em: <https://www.mathworks.com/help/images/ref/im2bw.html?s_tid=doc_ta > Acesso em: 10/06/2019

[3] MATLAB - Identificação de objetos redondos, 2010. [Internet] Disponível em: <https://la.mathworks.com/help/images/examples/identifying-round-objects.html> Acesso em: 04/08/2019

[4] XU, Lei; OJA, Erkki; KULTANEN, Pekka. A new curve detection method: randomized Hough transform (RHT). Pattern recognition letters, v. 11, n. 5, p. 331-338, 1990.

[5] MATLAB -Imfindcircles, 2010. [Internet] Disponível em: <https://www.mathworks.com/help/images/ref/imfindcircles.html?s_tid=doc_ta\#d117e127624> Acesso em: 10/06/2019 